\title{
Local demodulation of holograms using the Riesz transform with application to microscopy
}

\author{
Chandra Sekhar Seelamantula, ${ }^{1, *}$ Nicolas Pavillon, ${ }^{2,3}$ Christian Depeursinge, ${ }^{2}$ and Michael Unser ${ }^{3}$ \\ ${ }^{1}$ Department of Electrical Engineering, Indian Institute of Science, Bangalore 560 012, India \\ ${ }^{2}$ Microvision and Microdiagnostics Group (MVD), Ecole Polytechnique Fédérale de Lausanne (EPFL), \\ Lausanne CH 1015, Switzerland \\ ${ }^{3}$ Biomedical Imaging Laboratory, School of Engineering, Ecole Polytechnique Fédérale de Lausanne (EPFL), \\ Lausanne CH 1015, Switzerland \\ *Corresponding author: chandra.sekhar@ieee.org
}

Received January 26, 2012; revised July 13, 2012; accepted July 17, 2012;

posted August 23, 2012 (Doc. ID 162126); published September 17, 2012

\begin{abstract}
We propose a Riesz transform approach to the demodulation of digital holograms. The Riesz transform is a higherdimensional extension of the Hilbert transform and is steerable to a desired orientation. Accurate demodulation of the hologram requires a reliable methodology by which quadrature-phase functions (or simply, quadratures) can be constructed. The Riesz transform, by itself, does not yield quadratures. However, one can start with the Riesz transform and construct the so-called vortex operator by employing the notion of quasi-eigenfunctions, and this approach results in accurate quadratures. The key advantage of using the vortex operator is that it effectively handles nonplanar fringes (interference patterns) and has the ability to compensate for the local orientation. Therefore, this method results in aberration-free holographic imaging even in the case when the wavefronts are not planar. We calibrate the method by estimating the orientation from a reference hologram, measured with an empty field of view. Demodulation results on synthesized planar as well as nonplanar fringe patterns show that the accuracy of demodulation is high. We also perform validation on real experimental measurements of Caenorhabditis elegans acquired with a digital holographic microscope. (c) 2012 Optical Society of America
\end{abstract}

OCIS codes: $\quad 090.1995,100.2000,100.3010,100.3175,120.5050,180.3170$.

\section{INTRODUCTION}

The Hilbert transform is a powerful tool in modulation and communication theory [1] ]. It is central to the notion of Gabor's analytic signal formalism [2] and plays a vital role in optics as well as signal processing $[3,4]$. The Hilbert transform is linear, shift invariant, and unitary. It is associated with an antisymmetric phase response: for positive frequencies, it retards the phase by $\frac{\pi}{2}$, and for negative frequencies, it advances by $\frac{\pi}{2}$. The magnitude response is constant across all frequencies. These properties allow for mapping cosine functions into sines - a feature that has been extensively used for generating quadrature-phase signals and thereby for performing demodulation of amplitude and phase/frequency-modulated signals.

Several higher-dimensional extensions of the Hilbert transform have been proposed, which can be broadly grouped as follows depending on the specific properties of the associated kernel: (i) steerable extension based on the Riesz transform [5] and (ii) separable extensions based on the quarter-plane and half-plane geometries [6], which are not rotation covariant. Thanks to its steerability property [7,8], the Riesz transform can perform a Hilbert-transform-like analysis along any desired orientation [9], while the separable extensions are biased toward the horizontal or vertical directions. There is also another version of the analytic signal-due to Bülow and Sommerthat starts with separable Hilbert-transform constituents and combines them into four quadrature components using sophisticated quaternion algebra [10]. In this paper, we consider the steerable extension, namely, the Riesz transform, and then consider its complexified version, which we shall refer to as the complex Riesz transform. The complex Riesz transform was introduced by Larkin et al. to the optics community under the name of spiral-phase quadrature transform [11,12]. In the same year, Felsberg and Sommer introduced the Riesz transform to the signal processing community and also defined the monogenic signal as a two-dimensional (2D) counterpart of the analytic signal [13]. The Riesz transform is a vector operator in the sense that it takes a function and maps it into a vector of functions, whereas the quarter- and half-plane Hilbert transforms are scalar operators (they map functions into functions).

For the application part, we consider the fringe demodulation problem that arises in the context of digital holographic microscopy (DHM), which is a coherent imaging modality successfully used for biomedical imaging applications. The key idea behind digital holography is to digitally record a modulated wavefront. Its main advantage is to give access to the phase of the complex wave field, which cannot be measured directly otherwise. During reconstruction, the holograms are first demodulated and then brought into focus by using numerical techniques such as the Fresnel wave-propagation technique. The standard Fourier-based approach assumes a globally planar reference wave so that demodulation can be performed without causing distortions simply by performing frequency-domain translation. It is also necessary to compensate for the curvature of the reference wave before performing demodulation and Fresnel propagation. In state-of-the-art hologram reconstruction techniques, such compensation is performed by fitting a $2 \mathrm{D}$ phase function to a manually selected region of the hologram [14]. In the absence of phase compensation, the reconstructions 
suffer from severe amplitude and phase distortions. A related reconstruction approach is that of Liebling et al. [15], who perform a local quadratic fit to the hologram and estimate the object wave using an auxiliary variable method. Their method consists of solving a system of three linear equations at every pixel location. Liebling et al. reported their algorithm for planar reference waves, but it may be suitably extended to handle nonplanar reference waves as well. In a recent publication [16], we established a link between exact hologram demodulation and quarter-plane Hilbert transform under certain conditions specifically for the case of planar wavefronts.

The problem that we are interested in solving in this paper is that of hologram demodulation when the fringes are not planar. We propose a complex Riesz transform approach for hologram demodulation as an alternative to the more standard Fourier algorithms. The complex Riesz transform is a consistent generalization of the Hilbert transform in higher dimensions in the sense that its frequency-domain specification coincides with that of the Hilbert transform when the number of dimensions is reduced to one. We show that the onedimensional (1D) demodulation ideas based on the Hilbert transform are transposable to the $2 \mathrm{D}$ setup provided that we can properly estimate the (local) spatial orientation (the orientation map is essentially a $2 \mathrm{D}$ array whose entries are the angles of local orientation of the fringes at each location) of the modulating plane wave. One can then deploy the Riesz transform to compute a directional analog of the Hilbert transform (perpendicular to the wavefront of the modulating wave) to obtain a directional analytic signal. To implement the scheme, one needs a calibration step wherein a hologram is recorded with an empty field of view to get the local orientation and reference phase at each spatial location in the image. The main point is that the scheme remains applicable in the case when the modulation varies slowly across the field of view (for example, curved wavefronts) to the extent that it can be considered to be locally planar. We shall justify the approach based on a quasi-eigenfunction approximation [17]. Our treatment based on the quasi-eigenfunction approximation is different from the stationary-phase analysis of Larkin [12]. Whereas Larkin's approach is based on the evaluation of asymptotic integrals, our approach primarily relies on the approximate local planarity of the fringes, which is a more realistic condition. Moreover, the asymptotic behavior of the convolution integral (for example, as the frequency of the fringe tends to infinity) has limited practical significance. Since the Riesz transform adapts to the curvature in the fringes, the requirement of planar fringes is relaxed. Therefore, this approach also provides for automatic compensation of aberrations that arise due to curvature mismatch between the reference and object arms of a holography system. We note that it is not mandatory to have planar fringes in holography, but in order to achieve high-quality imaging, the curvature of the fringes must be taken into account in the demodulation procedure; otherwise, it would result in phase aberrations. With the proposed Riesz transform approach, curvature of the fringes is automatically compensated for.

\section{A. Contributions of the Paper}

We next list the contributions of this paper:

- A systematic development of the demodulation algorithm first in $1 \mathrm{D}$ and then in $2 \mathrm{D}$. The $1 \mathrm{D}$ formalism helps in appreciating the properties that a demodulation operator should possess and guides the design of the $2 \mathrm{D}$ operators.

- A quasi-eigenfunction approach to analyze the effect of the complex Riesz transform on modulated functions. This development leads to the vortex operator in a natural way.

- A reference hologram method to compute the orientation of the reference wave using the Riesz transform (the calibration step).

- The application and evaluation of the proposed Riesz demodulator on synthesized data as well as on holographic microscopy data.

\section{B. Organization of the Paper}

This work relies on a mix of signal processing and coherent optics techniques that may not necessarily be known to this readership. Accordingly, we have tried to make the presentation as self-contained as possible. The relevant aspects of hologram recording and reconstruction are presented briefly in Section 2. The quasi-eigenfunction approximation is presented in Section 3. We then address the hologram demodulation problem in $1 \bar{D}$, primarily for systematic development and for motivating the developments in the $2 \mathrm{D}$ case. We highlight the properties of the 1D Hilbert transform and show how it can be applied to compute the quadratures (Sections 4 and 5). This approach of starting with $1 \mathrm{D}$ and then moving to $2 \mathrm{D}$ capitalizes on the similarities and also brings up new issues related to orientation estimation, which do not exist in $1 \mathrm{D}$. Another key difference is that the Bedrosian/NuttallBedrosian theorems, which play a key role in 1D Hilbert transforms, do not have direct counterparts in 2D. Notwithstanding this deficiency, we show how 2D quadratures may be constructed using the Riesz transform (Section 6) and develop the associated demodulation technique (Section 7). We also present results on synthesized and experimental DHM data.

\section{Notations}

We shall work mainly with 2D functions, which are defined either in space or in frequency with the associated arguments being $\boldsymbol{x}=(x, y)$ or $\boldsymbol{\omega}=\left(\omega_{x}, \omega_{y}\right)$, respectively. The Fourier transform operator is denoted by $\mathcal{F}$. Its definition in $1 \mathrm{D}$ or $2 \mathrm{D}$ applies depending on the domain of definition of the functions involved. The wave vector is denoted as $\boldsymbol{k}=\left(k_{x}, k_{y}\right)$, where $k_{x}$ and $k_{y}$ are the wavenumbers in the $x$ and $y$ directions, respectively. The inner product of two index vectors is defined in the usual sense and is denoted as $\langle\cdot, \cdot\rangle$; specifically, $\langle\boldsymbol{k}, \boldsymbol{x}\rangle=k_{x} x+k_{y} y$. Two-dimensional sequences are represented using square bracket notation: for example, $\psi_{i}[\boldsymbol{m}]$ has the argument $\boldsymbol{m}=(m, n)$

\section{DIGITAL HOLOGRAM RECORDING AND RECONSTRUCTION}

\section{A. Hologram Recording}

A hologram is formed as a result of the interference between two mutually coherent waves-one emanating from the object, denoted by $o(\boldsymbol{x})$, and the other a reference wave $r(\boldsymbol{x})$. The interference pattern has a spatial intensity distribution $i(x)$, which is actually the hologram, given as

$$
\begin{aligned}
i(\boldsymbol{x}) & =|r(\boldsymbol{x})+o(\boldsymbol{x})|^{2} \\
& =|r(\boldsymbol{x})|^{2}+|o(\boldsymbol{x})|^{2}+r^{*}(\boldsymbol{x}) o(\boldsymbol{x})+r(\boldsymbol{x}) o^{*}(\boldsymbol{x}) .
\end{aligned}
$$




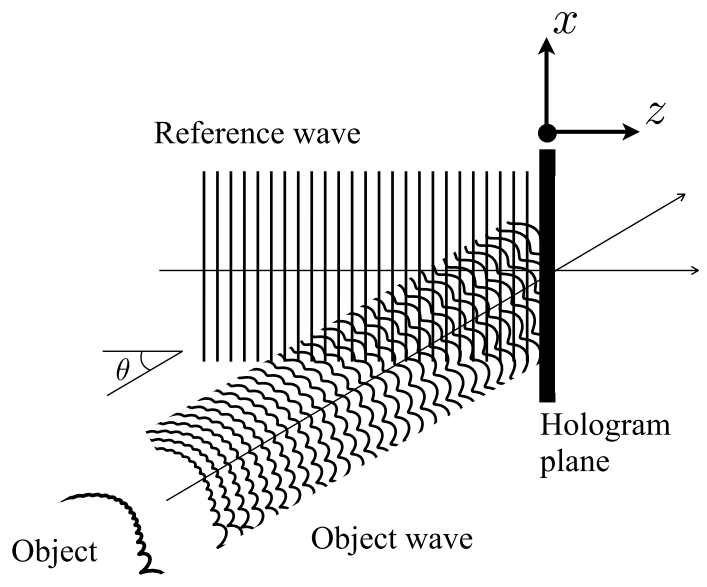

Fig. 1. Off-axis digital holography—recording.

The terms $|r(x)|^{2}$ and $|o(x)|^{2}$ are the intensities of the reference and object waves, respectively. The hologram $i(\boldsymbol{x})$ is suitably recorded at the hologram plane. In classical holography, a photographic plate is used to record the hologram. In stateof-the-art digital holography systems, a digital acquisition device such as a charge-coupled device camera placed at the hologram plane captures the spatial intensity distribution $[18,19]$. In off-axis holography, the two waves are separated by an angle $\theta$, as shown in Fig. 1 ; this configuration presents certain advantages for hologram reconstruction [20].

\section{B. Hologram Reconstruction}

Consider the reference to be a unit-modulus phase function: $r(\boldsymbol{x})=e^{j \phi(x)}$. To reconstruct the hologram, a plane wave $u(x)$ (illumination wave) is employed to illuminate the hologram, which creates a field $\psi_{o}(\boldsymbol{x})$ given as

$$
\begin{aligned}
\psi_{o}(\boldsymbol{x}) & =u(\boldsymbol{x}) i(\boldsymbol{x}) \\
& =\underbrace{u(\boldsymbol{x})+u(\boldsymbol{x})|o(\boldsymbol{x})|^{2}}_{\text {zero-order terms }}+\underbrace{u(\boldsymbol{x}) r^{*}(\boldsymbol{x}) o(\boldsymbol{x})+u(\boldsymbol{x}) r(\boldsymbol{x}) o^{*}(\boldsymbol{x})}_{\text {imaging terms }} .
\end{aligned}
$$

The zero-order functions modify the amplitude of $u(x)$, but not its phase, whereas the imaging terms contain the complex wave-field information. The spatial locations of the three functions are shown in Fig. 2. The off-axis configuration causes a separation between the imaging terms and the zero order.

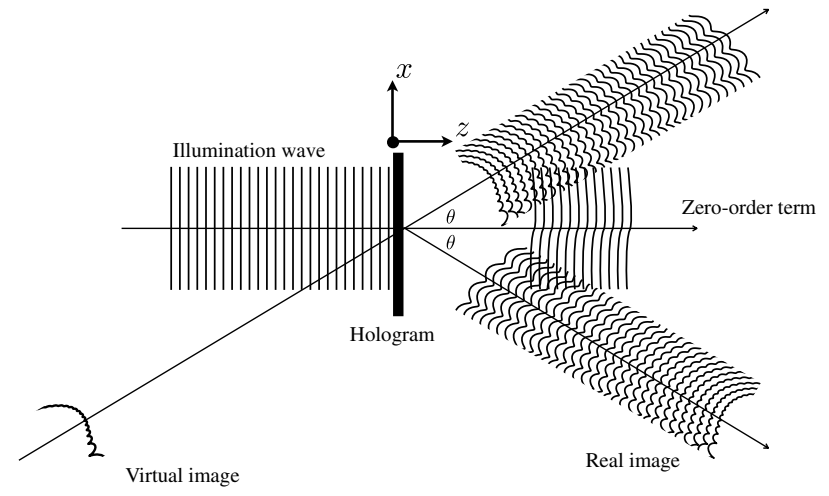

Fig. 2. Off-axis digital holography-reconstruction.
In digital reconstruction, the wave-propagation mechanism is replaced by a numerical approximation up to the second order, which leads to the so-called Fresnel propagation algorithm. In this case, the desired imaging order is first selected by Fourier filtering (illustrated in Fig. $\underline{8}$ below), which is then demodulated and Fresnel propagated to bring the complex wave field into focus.

The demodulation part is relatively easy if there are no optical aberrations and the reference is a plane wave. Optical aberrations are typically compensated for by using a numerical parametric lens approach, where the aberrated wave field is multiplied with a suitably defined phase mask [21,22]. Another approach to compensate for aberrations consists of employing a hologram with an empty field of view, known as the reference correction hologram $(\mathrm{RCH})$. Such a hologram contains the phase terms induced by the optical elements of the system. The aberration-corrected wave field is obtained by dividing the phase of the complex wave field with that of the $\mathrm{RCH}[14]$.

\section{QUASI-EIGENFUNCTION APPROXIMATION}

We know from linear system theory that complex exponentials are eigenfunctions of linear, shift-invariant (LSI) systems. To elucidate further, consider an LSI system with a point-spread function $h(x)$, characterized by its frequency response $\hat{h}(\omega)$. When excited with the complex exponential $e^{j\left\langle\omega_{c}, x\right\rangle}$, the system produces a response $e^{j\left\langle\omega_{c}, x\right\rangle} \hat{h}\left(\omega_{c}\right)$. Thus, for complex exponential inputs, the LSI system acts as a complex multiplier - this is the eigenfunction property. If the input has both frequency and amplitude modulations, then the eigenfunction property does not hold. However, one can make an approximation by assuming that the AMs and FMs are mild, that is, by assuming that their derivatives are of small magnitude over the support of $h$. Under this condition, the response of the LSI system to $o(\boldsymbol{x}) e^{j \phi(x)}$ is approximately given by $o(\boldsymbol{x}) e^{j \phi(x)} \hat{h}(\nabla \phi(\boldsymbol{x}))$, where $\nabla$ denotes the $2 \mathrm{D}$ gradient operator - this is referred to as the quasi-eigenfunction approximation (summarized in Fig. 3) and has been used in analyzing the effect of filtering on frequencymodulated signals in radio-frequency communication [23] and for deriving multidimensional energy operators in image processing [17]. If $\phi(x)=\left\langle\omega_{c}, x\right\rangle$, then $\nabla \phi(x)=\omega_{c}$, whereas if $\phi(x)=\left\langle\boldsymbol{\omega}_{c}, \boldsymbol{x}\right\rangle+\varphi(\boldsymbol{x})$, where $\varphi(\boldsymbol{x})$ is a mild modulation as considered in this paper, then it becomes necessary to invoke the quasi-eigenfunction approximation.

\section{QUADRATURES IN 1D}

We shall briefly recall some key properties of the Hilbert transform that are important to the developments in this paper. For a detailed treatment on Hilbert transforms, we refer the reader to $[\underline{3}, \underline{4}]$.

(a)

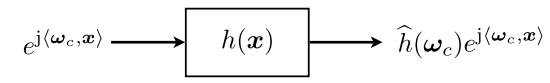

(b)

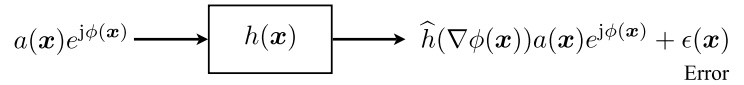

Fig. 3. Illustration of the (a) eigenfunction property and (b) quasieigenfunction approximation for LSI systems. The error $\epsilon(\boldsymbol{x})$ can be bounded in some suitable norm for well-behaved $h(x)$. 


\section{A. Hilbert Transform}

Definition: The Hilbert transform $\mathcal{H}$ is LSI and is specified by the transfer function $\hat{h}(\omega)=-j \operatorname{sign}(\omega)=-j \frac{\omega}{|\omega|}, \omega \in \mathbb{R}$. It is a unity-gain all-pass filter, associated with the phase function $-\frac{\pi}{2} \operatorname{sign}(\omega)$. The impulse response and frequency response are shown in Fig. 4. Because of its characteristic frequency response, a Hilbert transformer is also referred to as a phase shifter in communication theory [1]. By virtue of this property, it maps cosine functions into sine functions (the Hilbert-transform operation must be interpreted in the distributional sense for cosines and sines since they do not have finite energy):

$$
\mathcal{H}\left\{\cos \left(\omega_{c} \cdot+\theta\right)\right\}(x)=\sin \left(\omega_{c} x+\theta\right) .
$$

For finite-energy functions, the Hilbert transform is a unitary operator; that is, it preserves energy, or equivalently the $L^{2}(\mathbb{R})$ norm: $\|\mathcal{H} f(x)\|=\|f(x)\|$.

Given a finite-energy function $f(x)$, its analytic counterpart is defined as

$$
\begin{aligned}
f_{\text {as }}(x)= & f(x)+j \mathcal{H} f(x), \\
= & \underbrace{\sqrt{f^{2}(x)+(\mathcal{H} f)^{2}(x)}}_{\text {amplitude modulation (AM) }} \\
& \times \exp (j \underbrace{\tan ^{-1}\left(\frac{\mathcal{H} f(x)}{f(x)}\right)}_{\text {phase modulation (PM) }}) \text { (polar form). }
\end{aligned}
$$

The derivative of the PM is the frequency modulation (FM). The polar representation is important because it gives rise to an interpretation of a signal in terms of its AM and FM constituents. In practice, the phase is computed using the four-quadrant arc tangent (function atan2 in MATLAB), which gives angles in the range $[-\pi,+\pi]$.

\section{B. Product Theorems}

Under some conditions, the Hilbert transform of the product of two functions exhibits certain separability properties. We recall three product theorems, which are important for $1 \mathrm{D}$ demodulation:

Theorem 1 (Bedrosian [24]). If $f(x)$ and $g(x)$ are two square-integrable functions such that $f(x)$ has a Fourier spectrum band-limited to $\omega_{b}$, and $g(x)$ has a Fourier spectrum that is zero over $|\omega|<\omega_{b}$, that is, it is a high-pass signal, then $\mathcal{H}\{f g\}(x)=f(x) \mathcal{H} g(x)$.

Based on a calculation by Nuttall on the accuracy of the quadrature approximation of the Hilbert transform [25], Bedrosian gave a generalized version.

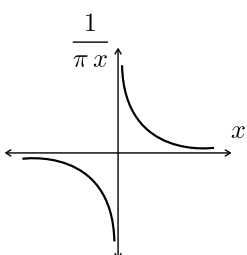

(a)

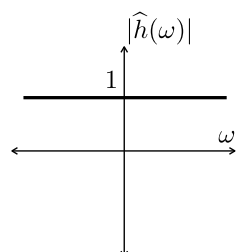

(b)

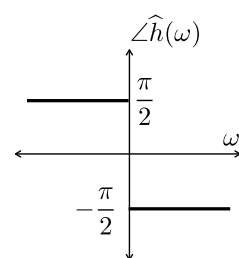

(c)
Fig. 4. Characterization of the Hilbert transformer: (a) impulse response, (b) magnitude spectrum, and (c) phase spectrum.
Theorem 2 (Bedrosian [25]). If $f(x)$ and $g(x)$ are two square-integrable functions such that the Fourier spectrum of $f(x)$ vanishes for $\omega<-\omega_{c}$ and that of $g(x)$ vanishes for $\omega>\omega_{c}$, then $\mathcal{H}\{f g\}(x)=f(x) \mathcal{H} g(x)$.

An interesting corollary of Theorem 2 follows; it specifies the conditions under which the Hilbert transform agrees with the quadrature model.

Corollary 1 (Nuttall-Bedrosian [25]). If the Fourier spectrum of $f(x)=o(x) e^{j \phi(x)}$ vanishes for $\omega<-\omega_{c}$, then $\mathcal{H}\left\{o(x) \cos \left(\omega_{c} x+\phi(x)\right)\right\}=o(x) \sin \left(\omega_{c} x+\phi(x)\right)$.

A product theorem related to amplitude-modulated sinusoids is stated next. It is an extension of Eq. (3) to amplitudemodulated cosines, provided that the maximum frequency in the $\mathrm{AM}$ is less than the carrier frequency.

Theorem 3 (Urkowitz [26]). If $o(x)$ is a square-integrable function band-limited to $\left[0, \omega_{b}\right]$ and $\omega_{b}<\omega_{c}$, then $\mathcal{H}\left\{o(x) \cos \left(\omega_{c} x+\theta\right)\right\}=o(x) \sin \left(\omega_{c} x+\theta\right)$.

\section{1D HOLOGRAM DEMODULATION}

It is instructive to first solve the demodulation problem in 1D as it gives insights into the mechanics of the demodulation approach. We then develop the $2 \mathrm{D}$ demodulation algorithm analogously.

According to the hologram formation model reviewed in Subsection 2.A, the 1D hologram is given by

$$
f(x)=\left|1+o(x) e^{-j \phi(x)}\right|^{2},
$$

where $\phi(x)$ is the instantaneous phase of the reference wave and the reference is assumed to be normalized, that is, $|r(x)|=1$. To proceed with the demodulation, we consider two cases: (i) linear phase, $\phi(x)=\omega_{c} x$, where we have exact results; and (ii) generalized phase, $\phi(x)$, where approximate analysis is carried out. The approximations arise mainly because product theorems are not applicable to generalized phase functions.

A. Linear Instantaneous Phase: $\phi(x)=\omega_{c} x$ In this case, we have $f(x)=\left|1+o(x) e^{-j \omega_{c} x}\right|^{2}$, where $\omega_{c}$ is the carrier frequency and $o(x)$ is the complex object wave with spectral support $\left[-\omega_{a}, \omega_{b}\right]$. By developing the squares, we get

$$
f(x)=1+|o(x)|^{2}+2 \operatorname{Re}\left\{o(x) e^{-j \omega_{c} x}\right\},
$$

where $\operatorname{Re}\{\cdot\}$ corresponds to the real part. The zero-order term $1+|o(x)|^{2}$ is supported over $\omega \in\left[-\left(\omega_{a}+\omega_{b}\right),\left(\omega_{a}+\omega_{b}\right)\right]$, whereas the modulated component $2 \operatorname{Re}\left\{o(x) e^{-j \omega_{c} x}\right\}$ is supported over two spectral regions specified together as $\left[-\omega_{c}-\omega_{b},-\omega_{c}+\omega_{a}\right] \cup\left[\omega_{c}-\omega_{a}, \omega_{c}+\omega_{b}\right]$. The spectral occupancy of the various terms is shown in Fig. 5 . If $\omega_{c} \geq 2 \omega_{a}+\omega_{b}$, then the three spectra do not overlap with each other. Under this condition, the zero order can be suppressed and the desired order can be selected by bandpass filtering. Thus, the function of interest in Eq. (6) is $\tilde{f}(x)=2 \operatorname{Re}\left\{o(x) e^{-j \omega_{c} x}\right\}$. We next perform a calibration experiment with an empty field of view, that is, $o(x)=1$, which results in the measurement

$$
g(x)=2+\underbrace{2 \cos \left(\omega_{c} x\right)}_{r(x)} .
$$

The constant is suppressed by removing the mean. Suppressing the constant is important because the Hilbert transform 
exhibits singular behavior for constants. Applying the Hilberttransform operator to $r(x)$ yields $2 \sin \left(\omega_{c} x\right)$. The complex reference wave is then obtained as the analytic signal

$$
r_{\mathrm{as}}(x)=r(x)+j \mathcal{H} r(x)=2 e^{-j \omega_{c} x} .
$$

Next, consider the analytic signal of $\tilde{f}(x)$ :

$$
\begin{aligned}
\tilde{f}_{\text {as }}(x) & =\tilde{f}(x)+j \mathcal{H} \tilde{f}(x), \\
& =2 \operatorname{Re}\left\{o(x) e^{-j \omega_{c} x}\right\}+j \mathcal{H}\left\{\operatorname{Re}\left\{2 o(x) e^{-j \omega_{c} x}\right\}\right\}, \\
& =2 o(x) e^{-j \omega_{c} x}
\end{aligned}
$$

(Corollary 1 of Theorem 2). From Eqs. () and (9), we get that

$$
o(x)=\frac{\tilde{f}_{\mathrm{as}}(x)}{r_{\mathrm{as}}(x)},
$$

which is the object wave encoded in the hologram. It must be noted that we have not assumed $o(x)$ to be real valued. Therefore, the demodulation technique is applicable to complexvalued object waves. This feature makes it attractive and relevant for holography applications where complex object waves arise naturally and the phase of the object wave plays a key role in the reconstruction process. The key properties aiding demodulation are Eq. (3) and Theorem 3, which are central to the construction of quadratures. The in-phase and quadrature components are taken together to form the complex signal, which is required in the final stage of demodulation [Eq. (10)].

\section{B. Generalized Phase}

If the Fourier spectrum of $o(x) e^{j \phi(x)}$ vanishes for $\omega<-\omega_{c}$, then the Nuttall-Bedrosian property applies (Corollary 1). Since the quadrature in this case matches exactly that given by the Hilbert transform, exact demodulation is possible by following the procedure described in Subsection 5.A. Otherwise, it is possible to generate only approximate quadratures. This aspect is discussed next.

Let us assume that $o(x)$ is a smooth function with a rate of variation smaller than that of the carrier frequency $\omega_{c}$ and that the phase function $\phi(x)$ is of the form $\phi(x)=\omega_{c} x+\varphi(x)$, where $\varphi(x)$ is the nonlinear phase variation about the carrier frequency such that $\left|\frac{d \varphi(x)}{d x}\right| \ll \omega_{c}$. Under these assumptions, the function $o(x) e^{j \phi(x)}$ can be locally approximated as a

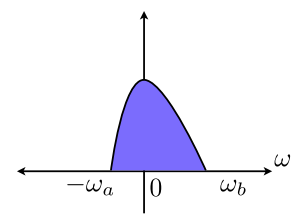

(a)

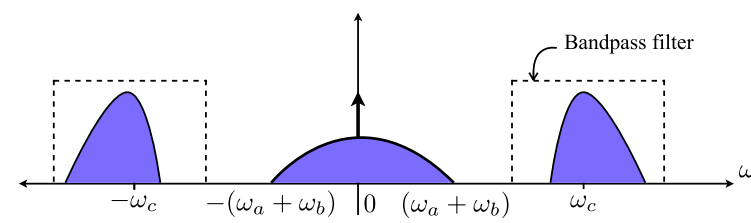

Fig. 5. (Color online) (a) Spectrum of $o(x)$ and (b) spectra of various functions in Eq. (6). The bandpass filter is used to select the desired imaging order. sinusoid. Invoking the quasi-eigenfunction approximation, we write

$$
\begin{aligned}
\mathcal{H}\left\{o(x) e^{j\left(\omega_{c} x+\varphi(x)\right)}\right\} & \approx \hat{h}\left(\omega_{c}+\frac{d \varphi(x)}{d x}\right) o(x) e^{j\left(\omega_{c} x+\varphi(x)\right)} \\
& =-j o(x) e^{j\left(\omega_{c} x+\varphi(x)\right)}
\end{aligned}
$$

Similarly,

$$
\begin{aligned}
\mathcal{H}\left\{o(x) e^{-j\left(\omega_{c} x+\varphi(x)\right)}\right\} & \approx \hat{h}\left(-\omega_{c}-\frac{d \varphi(x)}{d x}\right) o(x) e^{-j\left(\omega_{c} x+\varphi(x)\right)} \\
& =j o(x) e^{-j\left(\omega_{c} x+\varphi(x)\right)} .
\end{aligned}
$$

Combining Eqs. (11) and (12), we have the approximate quadrature relation:

$$
\mathcal{H}\left\{o(x) \cos \left(\omega_{c} x+\varphi(x)\right)\right\} \approx o(x) \sin \left(\omega_{c} x+\varphi(x)\right) .
$$

Nuttal and Bedrosian [25] showed that the approximation error in Eq. (13) has a finite energy, given by $\|\epsilon\|_{2}^{2}=$ $\frac{1}{\pi} \int_{-\infty}^{-\omega_{c}}\left|\mathcal{F}\left\{o(\cdot) e^{j \varphi(\cdot)}\right\}(\omega)\right|^{2} \mathrm{~d} \omega$, which is directly related to how far from being one sided the spectrum of $o(x) e^{j \phi(x)}$ is. This result indicates that, as $\omega_{c}$ increases, the quantity $\|\epsilon\|_{2}$ decreases and the Hilbert transform gives accurate estimates of the quadratures.

Having computed approximate quadratures, demodulation can then proceed in the same fashion as in the case of a constant-frequency carrier.

A proof of principle is shown in Fig. 6. We generated a complex object wave with real and imaginary parts as shown in Figs. 6(a) and 6(c), respectively. The FM is linear, sweeping from $\overline{0.4 \pi}$ radians to $0.5 \pi$ radians (normalized frequencies) over the observation duration of 1024 points. We find that, although the demodulation is approximate, the level of accuracy is acceptable. Note that the error cannot be made zero because of the approximations involved.

To recapitulate, the basic idea in the $1 \mathrm{D}$ algorithm is to obtain the quadrature components from the in-phase signals by exploiting the properties of the Hilbert transform. We pursue the same philosophy to derive the $2 \mathrm{D}$ demodulation algorithm.

\section{QUADRATURES IN 2D}

\section{A. Complex Riesz Transform}

The Riesz transform is a scalar-to-vector signal transformation specified by the mapping $f(x) \rightarrow f_{R}(x)$ as follows:

$$
f_{\boldsymbol{R}}(\boldsymbol{x})=\left(\begin{array}{l}
f_{1}(\boldsymbol{x}) \\
f_{2}(\boldsymbol{x})
\end{array}\right)=\left(\begin{array}{c}
\left(h_{x} * f\right)(x) \\
\left(h_{y} * f\right)(x)
\end{array}\right),
$$

where $\boldsymbol{x}=(x, y), f$ is the input image, and the filters $h_{x}$ and $h_{y}$ are characterized by their frequency responses as

$$
\hat{h}_{x}(\omega)=-j \frac{\omega_{x}}{\|\omega\|}, \quad \hat{h}_{y}(\omega)=-j \frac{\omega_{y}}{\|\omega\|} .
$$

To make an analogy, the Riesz transform is to the Hilbert transform what the gradient operator is to the derivative operator.

To derive the corresponding impulse responses $h_{x}(x)$ and $h_{y}(\boldsymbol{x})$, consider the Fourier transform pair $\mathcal{F}\left(\frac{1}{\|x\|}\right)=\frac{2 \pi}{\|\boldsymbol{\omega}\|}[\underline{8}]$, 

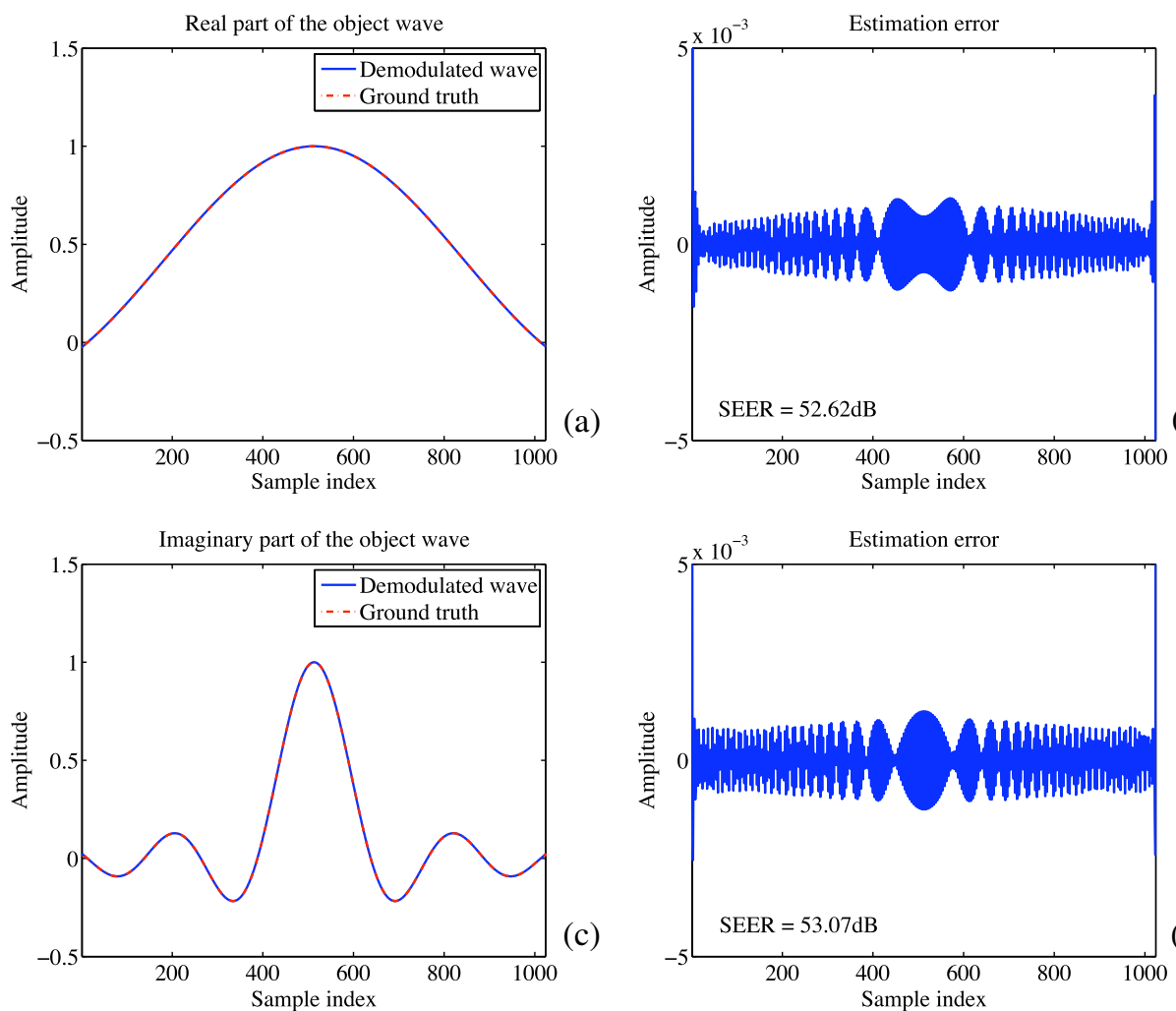

Fig. 6. (Color online) Performance of the Hilbert-transform-based demodulation algorithm. The AM is complex. The FM is linear, sweeping from $0.4 \pi$ radians to $0.5 \pi$ radians over the observation duration of 1024 points. The subplots (a) and (c) show the real and imaginary parts of the object wave, respectively, in comparison with the reconstructed ones. The subplots (b) and (d) show the associated estimation error. The signal-toestimation-error ratio (SEER) values are also indicated. Note that the demodulation accuracy is sufficient for most practical applications.

which is also a good example of circular symmetry of a 2D function and its Fourier transform. Performing partial differentiation with respect to $x$ or $y$, we obtain

$$
h_{x}(\boldsymbol{x})=\frac{x}{2 \pi\|\boldsymbol{x}\|^{3}}, \quad h_{y}(\boldsymbol{x})=\frac{y}{2 \pi\|x\|^{3}}
$$

In the present work, we construct a complexified version of the 2D Riesz operator (which we refer to as the complex Riesz transform), by combining the two Riesz components in Eq. (14), which correspond to two orthogonal directions, into a single complex function. The $2 \mathrm{D}$ complex Riesz operator, denoted by $\mathcal{R}$, is specified by its spectral behavior:

$$
\mathcal{R} f(\boldsymbol{x}) \stackrel{\mathcal{F}}{\leftrightarrow} \underbrace{\frac{-j \omega_{x}+\omega_{y}}{\|\omega\|}}_{\hat{h}_{\mathcal{R}}(\boldsymbol{\omega})} \hat{f}(\boldsymbol{\omega}),
$$

where $\hat{f}(\boldsymbol{\omega})=\mathcal{F}\{f\}(\boldsymbol{\omega})=\int_{\mathbb{R} \times \mathbb{R}} f(\boldsymbol{x}) e^{-j\langle\boldsymbol{\omega}, \boldsymbol{x}\rangle} \mathrm{d}^{2} \boldsymbol{x}$ is the 2D Fourier transform of $f$ and $\hat{h}_{\mathcal{R}}(\omega)=\left(\hat{h}_{x}+j \hat{h}_{y}\right)(\omega)=-j \frac{\omega}{\|\omega\|}=$ $e^{j \psi(\omega)}$ is the complex Riesz transfer function, where $\psi$ denotes the phase angle. The function $\hat{h}_{\mathcal{R}}(\omega)$ is a phase-only function with a unity magnitude and a phase response that resembles a spiral (see Fig. 7).

\section{B. Properties of the Complex Riesz Transform}

We recall from [9] some important properties of the complex Riesz transform that are relevant to the present discussion. The complex Riesz transform is a shift- and scale-invariant operator. It is also unitary, which follows directly from the property that $\left|\hat{h}_{\mathcal{R}}(\omega)\right|^{2}=1$. Therefore, it is also a self-adjoint operator; that is, $\mathcal{R}^{-1}=\mathcal{R}^{*}$, where

$$
\mathcal{R}^{*} f(\boldsymbol{x}) \stackrel{\mathcal{F}}{\leftrightarrow} \frac{j \omega_{x}+\omega_{y}}{\|\omega\|} \hat{f}(\omega) .
$$

The complex Riesz transform of a cosine wave of frequency $\boldsymbol{\omega}_{c}=\left(\omega_{c} \cos \theta_{0}, \omega_{c} \sin \theta_{0}\right)$ is given by

$$
\mathcal{R} \cos \left(\left\langle\omega_{c}, .\right\rangle\right)(\boldsymbol{x})=e^{j \theta_{0}} \sin \left(\left\langle\omega_{c}, \boldsymbol{x}\right\rangle\right)
$$

It is also closely related to the directional Hilbert transform as follows:

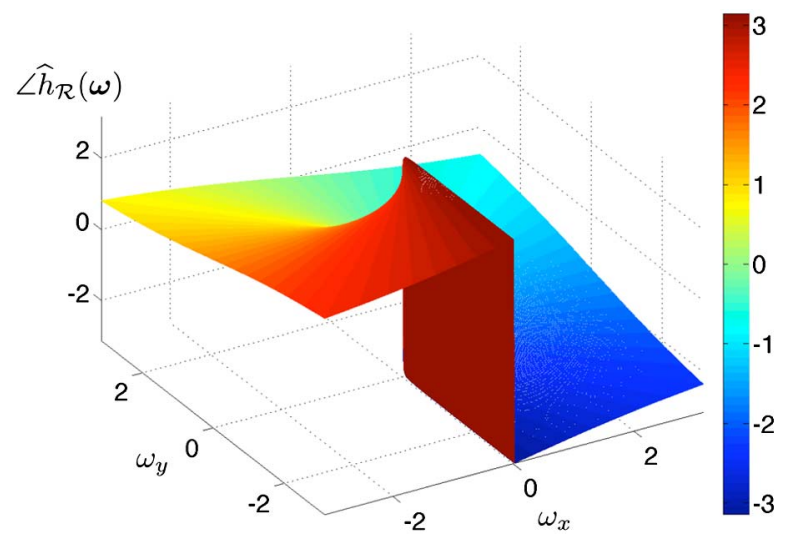

Fig. 7. (Color online) Phase response of the complex Riesz operator over the domain $[-\pi, \pi] \times[-\pi, \pi]$. The units for all three axes are radians. 


$$
\mathcal{H}_{\theta} f(x) \stackrel{\text { def }}{=} \cos \theta f_{1}(x)+\sin \theta f_{2}(x)=\operatorname{Re}\left\{e^{-j \theta} \mathcal{R} f(x)\right\},
$$

which can be established by taking into account the steerability of the Riesz transform [7,8]. By steerability, we mean that the transform computed along any direction can be expressed as a linear combination of the transforms computed along other directions. As a result, it suffices to compute the transform along those elementary directions (in this case, $x$ and $y$ ) and then use linear combinations to obtain the transform computed along any other direction (the linear combination weights depend on the desired orientation). For more details, the reader is referred to $[\underline{7}, \underline{8}]$. The manner in which the directional Hilbert transform and the Riesz transform are related is analogous to the fashion in which the directional derivative and the gradient operator are related.

By plugging Eq. (19) into Eq. (20), we get that

$$
\mathcal{H}_{\theta_{0}} \cos \left(\left\langle\omega_{c}, .\right\rangle\right)(x)=\sin \left(\left\langle\omega_{c}, x\right\rangle\right)
$$

If the direction for computing the Hilbert transform does not match the orientation of the cosine wave, we have that

$$
\mathcal{H}_{\theta} \cos \left(\left\langle\omega_{c}, .\right\rangle\right)(\boldsymbol{x})=\underbrace{\cos \left(\theta-\theta_{0}\right)}_{\text {roll-off }} \sin \left(\left\langle\omega_{c}, \boldsymbol{x}\right\rangle\right)
$$

The above equation is obtained by substituting Eq. (19) in Eq. (20) considering $\theta \neq \theta_{0}$. The $\cos \left(\theta-\theta_{0}\right)$ roll-off calls for precise orientation estimation in order to have high accuracy in computing directional Hilbert transforms. We next show how the orientation is computed using the Riesz transform in a structure-tensor formalism.

\section{Structure-Tensor Formalism for Estimating Orientation}

The structure-tensor formalism allows for precise calculation of local orientation. It gives accurate results for plane waves and for locally planar fringe patterns. The local orientation of a fringe at position $x_{0}$ is specified as the solution to the optimization problem:

$$
\theta\left(x_{0}\right)=\arg \max _{\alpha \in[-\pi, \pi]}\left(v *\left|\mathcal{H}_{\alpha} f\right|^{2}\right)\left(x_{0}\right),
$$

where $v$ is a positive, radially symmetric localizing function (typically, a symmetric Gaussian smoothing kernel is used) and the operator $\mathcal{H}_{\alpha}$ follows the definition given in Eq. (20). The cost function in Eq. (23) is rewritten as

$$
\begin{aligned}
\left(v *\left|\mathcal{H}_{\alpha} f\right|^{2}\right)\left(x_{0}\right)= & v *\left(\cos \theta f_{1}(\boldsymbol{x})+\sin \theta f_{2}(\boldsymbol{x})\right)^{2} \\
= & \cos ^{2} \theta\left(v * f_{1}^{2}\right)+\sin ^{2} \theta\left(v * f_{2}^{2}\right) \\
& +2 \cos \theta \sin \theta v *\left(f_{1} f_{2}\right) \\
= & \boldsymbol{u}^{T} \mathbf{J}\left(\boldsymbol{x}_{0}\right) \boldsymbol{u},
\end{aligned}
$$

where $\boldsymbol{u}=\left(\begin{array}{c}\cos \theta \\ \sin \theta\end{array}\right)$ and

$$
\mathbf{J}\left(\boldsymbol{x}_{0}\right)=\left(\begin{array}{cc}
v * f_{1}^{2} & v *\left(f_{1} f_{2}\right) \\
v *\left(f_{1} f_{2}\right) & v * f_{2}^{2}
\end{array}\right) .
$$

The optimum orientation is specified by the eigenvector corresponding to the maximum eigenvalue $\lambda_{\text {max }}$. The orientation $\theta$ is directly given in terms of the entries of the structure tensor $\mathbf{J}$ as follows:

$$
\theta\left(\boldsymbol{x}_{0}\right)=\frac{1}{2} \tan ^{-1}\left(\frac{2 v *\left(f_{1} f_{2}\right)}{v * f_{2}^{2}-v * f_{1}^{2}}\right) .
$$

\section{2D HOLOGRAM DEMODULATION}

As per the 2D hologram formation model, the measured intensity is given by $f(\boldsymbol{x})=\left|1+o(\boldsymbol{x}) e^{-j \phi(x)}\right|^{2}$, where $o(\boldsymbol{x})$ is the complex-valued object wave and $\phi(x)$ is the phase function of the reference wave. This model for the hologram is general and covers any fringe shape [straight if $\phi(x)$ is linear, and curved if $\phi(x)$ is quadratic, etc.] and can account for curvature of both object and reference beams.

Developing the squares, we get that

$$
f(x)=\underbrace{1+|o(x)|^{2}}_{\text {zero order }}+2 \operatorname{Re}\left\{o(x) e^{-j \phi(x)}\right\} .
$$

To assess the spectral occupancy of the various components in Eq. (26), we consider a plane-wave reference, that is, $\phi(x)=\left\langle\overline{\omega_{c}}, \boldsymbol{x}\right\rangle$, and an object wave that has a circular spectral support (see Fig. 8). The assumption of circular spectral support is compatible with the practical setting because the spectral influence of the microscope objective employed in the measurements indeed results in such spectra. Let the spectral radius of $o(x)$ be $\sigma$. Correspondingly, the spectral radius of the zero-order term is $2 \sigma$. The modulated diffraction orders can be separated from the zero order by an appropriate choice of $\omega_{c}$. Specifically, by selecting $\omega_{c}>\frac{3 \sigma}{\sqrt{2}}$, overlap between the zero order and the imaging orders is avoided. The zero order can then be suppressed and the desired order selected by bandpass filtering. Alternatively, the zero order can be suppressed by separately measuring $|o(\boldsymbol{x})|^{2}$, for example by blocking the reference arm illumination and then subtracting it from Eq. (26). However, in this case, it is not required to have a separation between the zero order and the imaging terms. Thus, effectively, the function of interest in Eq. (26) is $2 \operatorname{Re}\left\{o(\boldsymbol{x}) e^{-j \phi(x)}\right\}$.

For the demodulation problem, we consider two cases: (i) plane-wave reference and a constant $o(x)$ and (ii) nonplanar

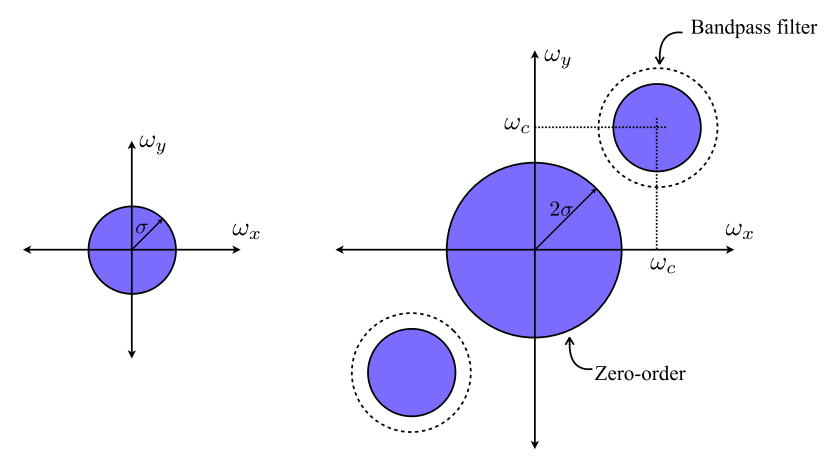

(b)

The right-hand side of Eq. (24) is a quadratic form, and optimizing it is equivalent to solving an eigenvalue problem [27].

Fig. 8. (Color online) (a) Spectral support of $o(\boldsymbol{x})$; (b) Spectral support of various functions in Eq. (26). The bandpass filter is used to suppress the zero-order terms. 
waves but with $\phi(x)$ and $o(x)$ assumed to be slowly varying. The first case helps in understanding the mechanics of demodulation, while the latter one is of greater practical relevance.

\section{A. $\phi(x)=\left\langle\omega_{c}, x\right\rangle$ and $o(x)=a$ (Constant)}

Consider the function of interest in Eq. (26): $\tilde{f}(x)=$ $2 \operatorname{Re}\left\{a e^{-j\left\langle\omega_{c}, x\right\rangle}\right\}$. As in the $1 \mathrm{D}$ demodulation algorithm, we first conduct a calibration experiment by choosing an empty field of view (that is, $o(x)=1$ ). The corresponding measurement is $g(\boldsymbol{x})=1+\underbrace{2 \cos \left(\left\langle\omega_{c}, \boldsymbol{x}\right\rangle\right)}_{r(\boldsymbol{x})}$. After suppressing the constant, the local orientation map of $r(x)$ is estimated by adopting the structure-tensor approach described in Subsection 6.C. Let the estimated orientation be denoted by $\hat{\theta}(x)$. The corresponding complex signal is obtained according to the directional Hilbert-transform method, wherein the orientation at each point is chosen to be orthogonal to that specified by $\hat{\theta}(\boldsymbol{x})$ :

$$
r_{\text {das }}(x)=r(x)+j \mathcal{H}_{\hat{\theta}(x)}\{r(x)\},
$$

where the subscript das indicates that it is a directional analytic signal. The directional Hilbert transform is related to the Riesz transform by virtue of the property stated in Eq. (20). Therefore, we have

$$
r_{\mathrm{das}}(\boldsymbol{x})=r(\boldsymbol{x})+j \operatorname{Re}\left\{e^{-j \hat{\theta}(\boldsymbol{x})} \mathcal{R} r(\boldsymbol{x})\right\} \approx 2 e^{-j\left\langle\omega_{c}, \boldsymbol{x}\right\rangle},
$$

where the error is of the order of $(\theta-\hat{\theta})^{2}$ [from the Taylor series approximation of the cosine roll-off in Eq. (22)]. The structure-tensor approach gives accurate orientation estimates for plane waves, and therefore the error is negligible. Similarly, the directional analytic signal corresponding to $\tilde{f}(x)$ is computed as

$$
\tilde{f}_{\text {das }}(\boldsymbol{x})=\tilde{f}(\boldsymbol{x})+j \operatorname{Re}\left\{e^{-j \hat{\theta}(x)} \mathcal{R} \tilde{f}(\boldsymbol{x})\right\} \approx 2 a e^{-j\left\langle\omega_{c}, \boldsymbol{x}\right\rangle} .
$$

For constant $o(x)$, the orientations associated with $\tilde{f}(x)$ and $r(x)$ are the same. Therefore, the constant-valued object wave is demodulated as

$$
a \approx \frac{\tilde{f}_{\text {das }}(x)}{r_{\text {das }}(x)} .
$$

\section{B. Slowly Varying $o(x)$ and $\theta(x)$}

In this case, we invoke the quasi-eigenfunction property (see Section 3) to specify the Riesz transform of $o(\boldsymbol{x}) e^{j \phi(x)}$ :

$$
\mathcal{R}\left\{o(\cdot) e^{j \phi(\cdot)}\right\}(\boldsymbol{x}) \approx o(\boldsymbol{x}) e^{j \phi(x)} \hat{h}_{\mathcal{R}}(\nabla \phi)(\boldsymbol{x}) .
$$

By applying the quasi-eigenfunction property to $o(x) e^{-j \phi(x)}$, we have that

$$
\mathcal{R}\left\{o(\cdot) e^{-j \phi(\cdot)}\right\}(\boldsymbol{x}) \approx o(\boldsymbol{x}) e^{-j \phi(\boldsymbol{x})} \hat{h}_{\mathcal{R}}(-\nabla \phi)(\boldsymbol{x}) .
$$

Combining Eqs. (31) and (32) yields the following:

$$
\mathcal{R}\{o(\cdot) \cos \phi(\cdot)\}(x) \approx j e^{j \psi(\nabla \phi(x))} o(x) \sin \phi(x),
$$

where $\psi$ is the phase angle associated with the Riesz transfer function (see Subsection 6.A). Equation (33) is then rearranged as follows:

$$
\underbrace{-j e^{-j \psi(\nabla \phi(x))} \mathcal{R}}_{\mathcal{V}}\{o(\cdot) \cos \phi(\cdot)\}(\boldsymbol{x}) \approx o(\boldsymbol{x}) \sin \phi(\boldsymbol{x}) .
$$

Note that the left-hand side of Eq. (34) is exactly equivalent to the so-called vortex operator of Larkin et al. [11,12], denoted by $\mathcal{V}$ acting on $o(\boldsymbol{x}) \cos \phi(\boldsymbol{x})$. Effectively, the vortex operator does the job of computing the Riesz transform and then compensating for the fringe orientation (which is equivalent to a directional Hilbert transform). The net effect is that the vortex operator is oriented along the local direction that is estimated from the reference using the structure tensor. It also changes the polarity of the spectral lobes associated with the localized fringe pattern, the effect of which is to convert a cosine pattern to a sine pattern. Thus, the key equation in the construction of $2 \mathrm{D}$ quadratures is the following:

$$
\mathcal{V}\{o(\cdot) \cos \phi(\cdot)\}(\boldsymbol{x}) \approx o(\boldsymbol{x}) \sin \phi(\boldsymbol{x}) .
$$

To make an analogy, the vortex operator is to $2 \mathrm{D}$ fringes what the Hilbert transform is to modulated 1D functions.

The quadrature corresponding to the case with $o(x)=1$ may be derived from Eq. (35). The directional analytic signal corresponding to $o(\boldsymbol{x}) \cos \phi(x)$ is

$$
\tilde{f}_{\text {das }}(\boldsymbol{x})=o(\boldsymbol{x}) \cos \phi(\boldsymbol{x})+j \mathcal{V}\{o(\cdot) \cos \phi(\cdot)\}(\boldsymbol{x}) \approx o(\boldsymbol{x}) e^{j \phi(\boldsymbol{x})}
$$

and that corresponding to $r(x)$ is

$$
r_{\mathrm{das}}(\boldsymbol{x})=\cos \phi(\boldsymbol{x})+j \mathcal{V}\{\cos \phi(\cdot)\}(\boldsymbol{x}) \approx e^{j \phi(x)} .
$$

The object wave is demodulated as follows:

$$
o(x) \approx \frac{\tilde{f}_{\text {das }}(x)}{r_{\text {das }}(x)}
$$

The analogy between the $1 \mathrm{D}$ and $2 \mathrm{D}$ demodulation procedures is illustrated in Fig. 9. Note that taking the modulus of $\tilde{f}_{\text {das }}(x)$ to obtain the $\mathrm{AM}$ is not suitable for holography since we are interested in complex-valued $o(\boldsymbol{x})$. A summary of the proposed methodology is as follows.

\section{Calibration}

(a) Estimation of local orientation map $\theta(x)$ of the reference hologram as specified in Eq. (25).

(b) Compute the local directional Hilbert transform and associated directional analytic signal at every $\boldsymbol{x}$ (using the operator $\mathcal{V}$ ) according to Eq. (37).

\section{Hologram Demodulation}

(a) From the hologram $i(\boldsymbol{x})$, suppress the zero-order terms either by bandpass filtering or by subtracting the holograms corresponding to $r(\boldsymbol{x})=0$ and $o(\boldsymbol{x})=0$ [as explained after Eq. (26)].

(b) Compute the directional Hilbert transform Eq. (36) based on the directionality information stored in the orientation map of step 1 . 


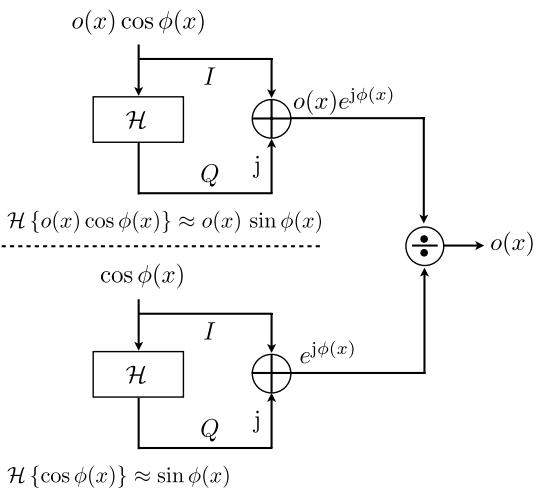

1-D demodulation

(a)

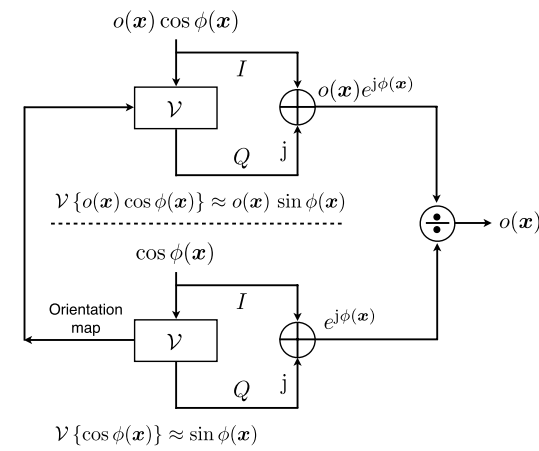

2-D demodulation

(b)

Fig. 9. Analogy between 1D and 2D demodulation: (a) Hilbert-transform-based demodulation of 1D signals and (b) Riesz-transform-based demodulation of 2D fringes. $I$, in-phase channel; $Q$, quadrature-phase channel (standard terminology in communication/modulation literature).

(c) Compute the complex object wave according to Eq. (38).

Next, the object wave is subjected to Fresnel propagation in order to bring the object into focus. This step is standard and is executed as in the case of standard Fourier reconstruction algorithms. Note that the main ingredient that allows for handling of local phase aberrations is the vortex operator $\mathcal{V}$ whose orientation is controlled adaptively using the reference orientation map.

\section{Simulation Results}

To validate the demodulation technique, we consider the modulation of the image "Peppers" on a constant-frequency plane wave. The results are shown in Fig. 10. We use apodization (windowing) for all images, which is a standard practice in holography mainly to mitigate the ripple at the boundaries of the image arising out of fast Fourier transform implementations (which implicitly assume a periodic image). The modulated image and the carrier are the inputs to the Riesz demodulator. The demodulated image and the Riesz structuretensor-based orientation map are also shown in the figure. The orientation is nearly constant, which is what we would expect to have. The difference between the original image and the demodulated image is displayed in Fig. 10(f). When comparing the result with the original image, we observe that the reconstruction error is small. The smooth regions are generally well reproduced, while some errors appear at the edges. This an expected phenomenon because edges are discontinuities that contain high-frequency components. In such regions, a clear separation between the modulation and the carrier is lacking,

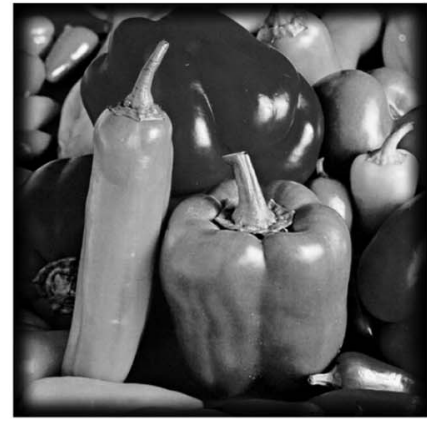

(a)

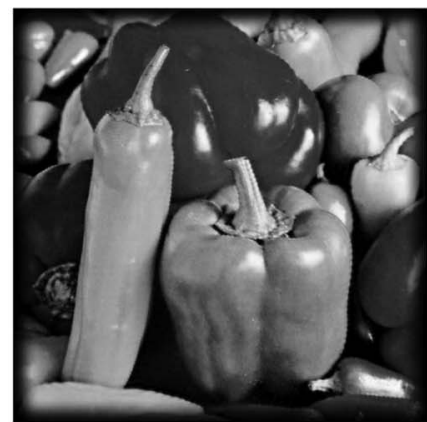

(d)

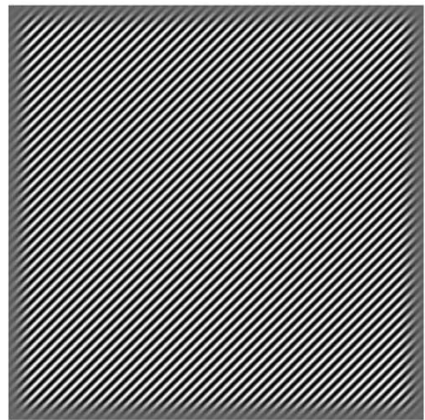

(b)

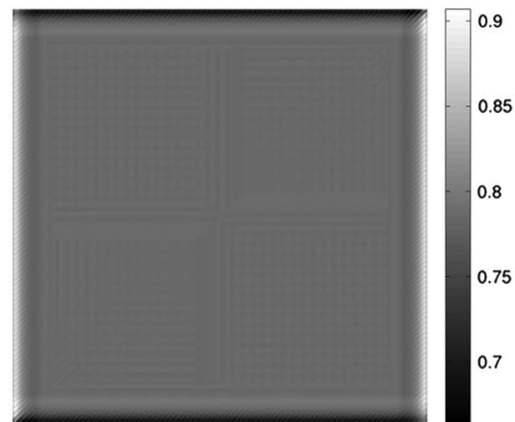

(e)

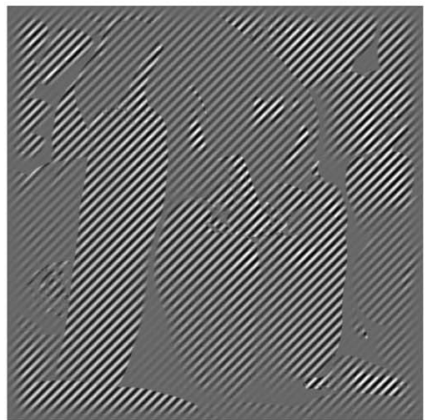

(c)

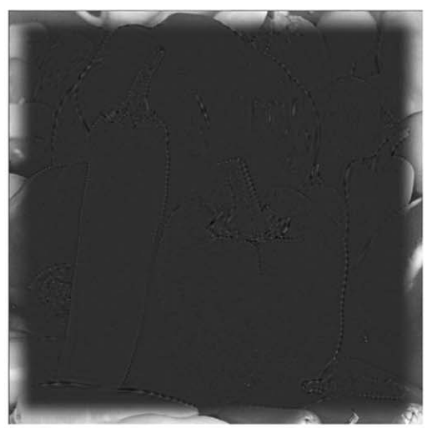

(f)

Fig. 10. Simulation results for linear-frequency modulation. (a) Original image, (b) carrier wave, (c) modulated image, (d) reconstructed image, (e) orientation map, and (f) reconstruction error. The orientation map is estimated with a Gaussian kernel of standard deviation $\sigma=5$. 
and hence the modulation model, strictly speaking, is not valid. Apart from that, the quality of demodulation is quite accurate.

We next repeated the experiment with a circular carrier wave of radially constant frequency. The results are displayed in Fig. 11. We observe that the Riesz demodulator is quite efficient at handling the curvature of the fringes because it effectively compensates for the orientation of the fringes and straightens them out, resulting in high-quality demodulation. The orientation map derived by the Riesz structure tensor is also quite accurate and globally smooth in spite of the fact that it is computed only locally.

\section{EXPERIMENTS ON REAL HOLOGRAMS}

We next validate the performance of the Riesz demodulator on real holograms of Caenorhabditis elegans (C. elegans). Brief descriptions of the specimen and the experimental setup follow.

\section{A. Specimen-C. elegans}

C. elegans is a multicellular eukaryotic organism that is studied as a model for a variety of reasons. Strains of $C$. elegans are inexpensive to breed and are suitable for long-term storage. C. elegans is transparent, facilitating the study of cellular differentiation and other developmental processes. C. elegans is one of the simplest organisms with a nervous system, whose connectivity pattern has been completely mapped. In particular, C. elegans is useful in the study of meiosis. It is also the first multicellular organism to have its genome completely sequenced. Fascinating pictures of C. elegans and information are available on the WORMATLAS (http://www.wormatlas.org/).

\section{B. Experimental Setup}

Since the fixed $C$. elegans specimen is transparent, the measurements were taken using a standard transmission holographic microscope [19]. The microscope objective has a magnification factor of 20 and a numerical aperture of 0.45 . The laser diode light source employed has a wavelength of $668 \mathrm{~nm}$. A Basler A102f camera with a pixel size of $6.45 \mu \mathrm{m}$ is employed for recording the holograms, with an exposure time of approximately $8 \mathrm{~ms}$. The holograms are of size $1024 \times$ 1024 pixels. We measured a reference hologram and a hologram with the sample. The reconstruction distance is approximately $7 \mathrm{~cm}$.

To validate the demodulation approach on curved fringes, we introduce a curvature mismatch between the reference and object beams by simply changing the position of a lens in the reference arm. The experimental results corresponding to the standard off-axis configuration are shown in Fig. 12, where the fringe curvature is barely identifiable due to the strong modulation; the results corresponding to the off-axis configuration with a small angle, sufficient to separate the imaging orders, are shown in Fig. 13, where the wavefront curvature mismatch can be identified in the fringe curvature and chirp. In both cases, the Riesz demodulator produces good-quality amplitude- and phase-contrast images. The phase image is particularly important because $C$. elegans gives rise to sharp phase-contrast images. Because of the nature of the specimen, the effect on the hologram generation consists in a local modification of the fringe orientation from the phase part of the signal, as can be seen particularly in Fig. 13(c).

One can appreciate the efficiency of the reconstruction in the flatness of the phase images [see Figs. 12(f) and 13(f)], as imprecisions in demodulation would readily induce visible changes in the phase profile. Furthermore, the phase-contrast

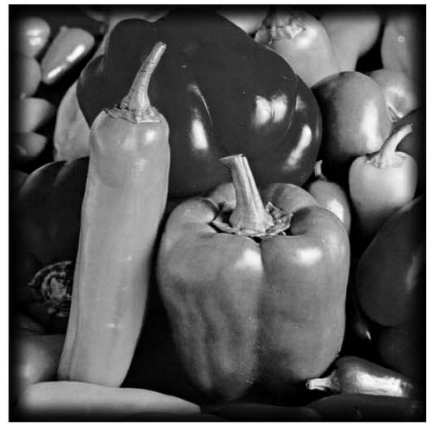

(a)

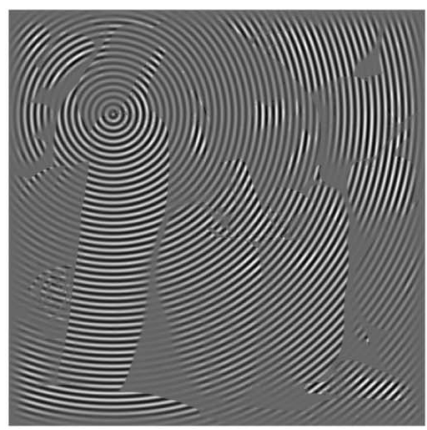

(d)

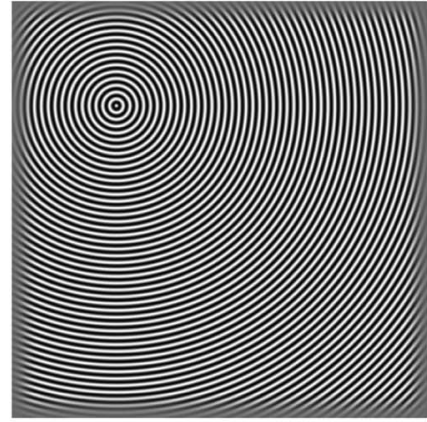

(b)

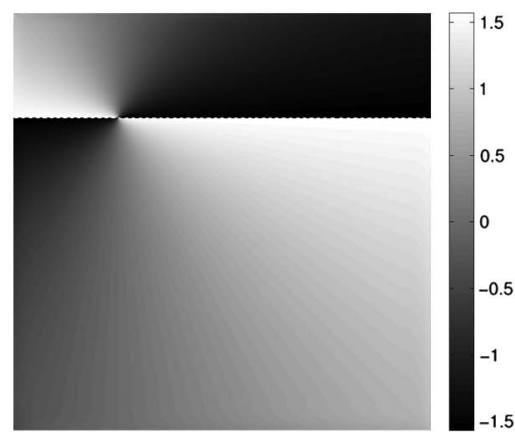

(e)

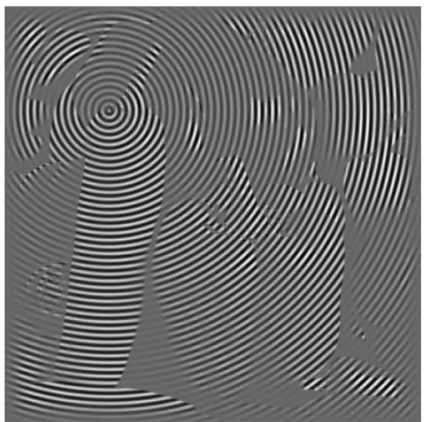

(c)

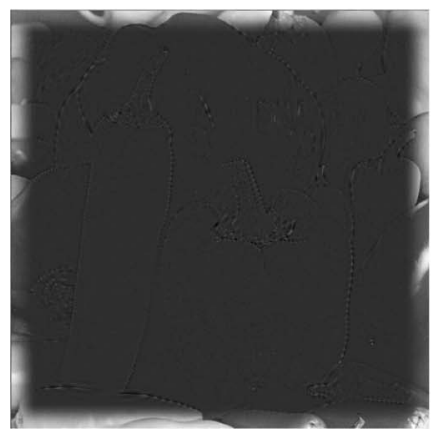

(f)

Fig. 11. Simulation results for circular-frequency modulation. (a) Original image, (b) carrier wave, (c) modulated image, (d) reconstructed image, (e) orientation map, and (f) reconstruction error. The orientation map is estimated with a Gaussian kernel of standard deviation $\sigma=5$. 


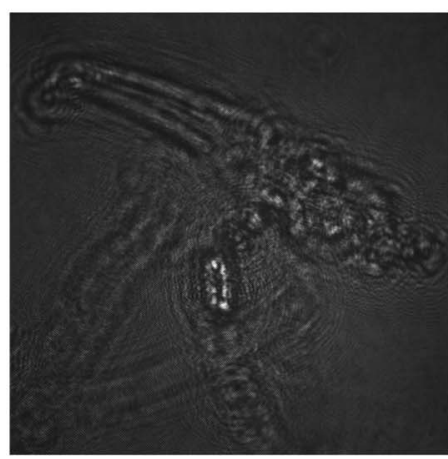

(a)

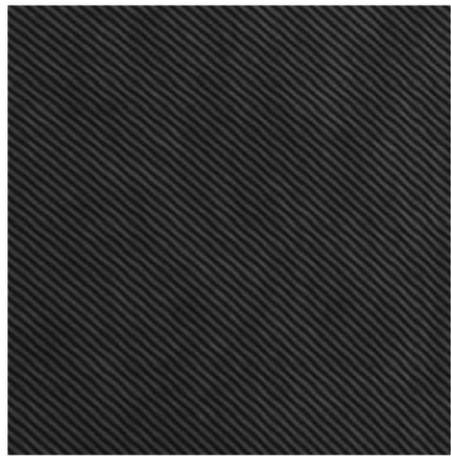

(d)

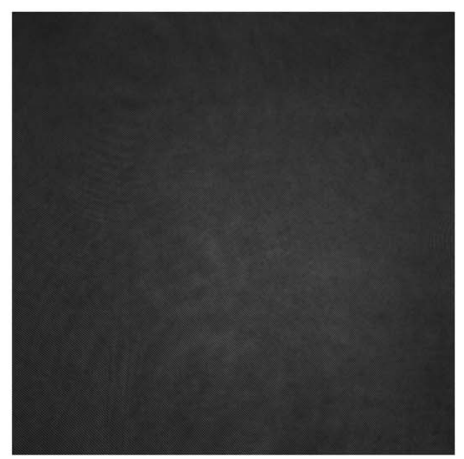

(b)

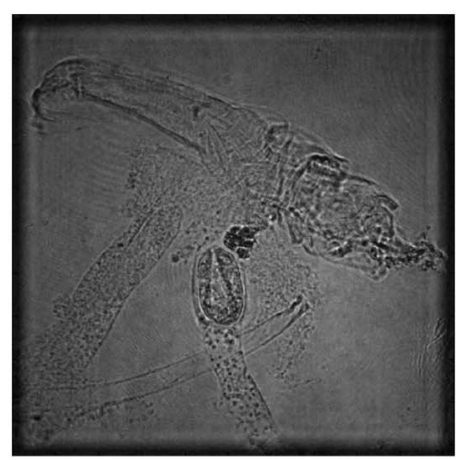

(e)

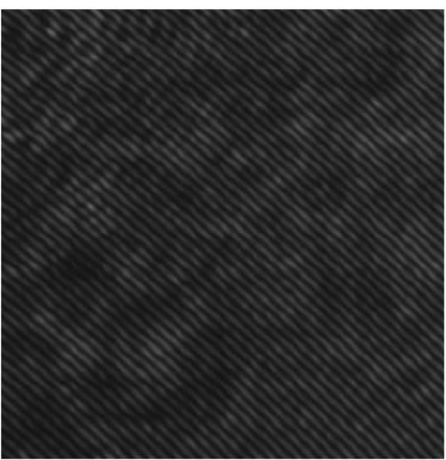

(c)

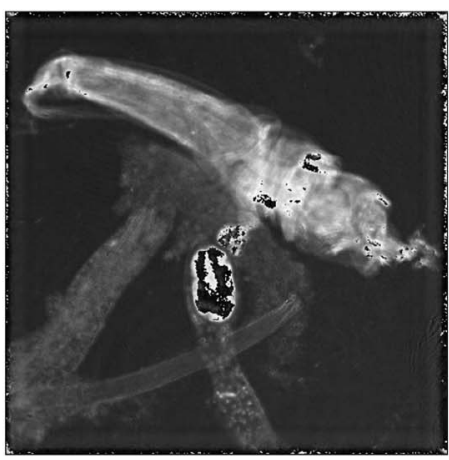

(f)

Fig. 12. Riesz demodulation performance on C. elegans holograms. (a) Hologram of C. elegans, (b) reference hologram, (c) zoomed-in part of the hologram (approximately 1/16th the size of the hologram from the lower left corner), (d) zoomed-in part of the reference hologram (approximately $1 / 16$ th the size of the reference hologram from the lower left corner, (e) amplitude-contrast image, and (f) phase-contrast image (wrapped phase). The orientation map is estimated with a Gaussian kernel of standard deviation $\sigma=5$.

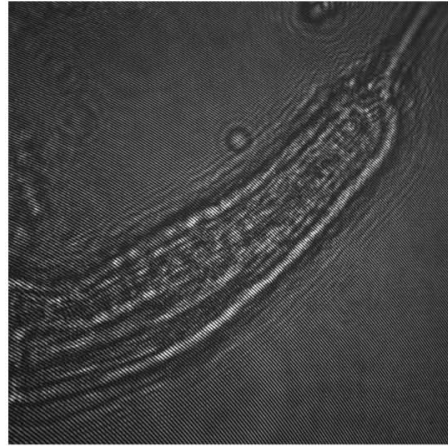

(a)

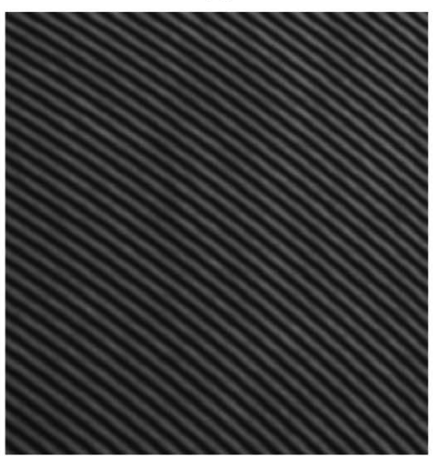

(d)

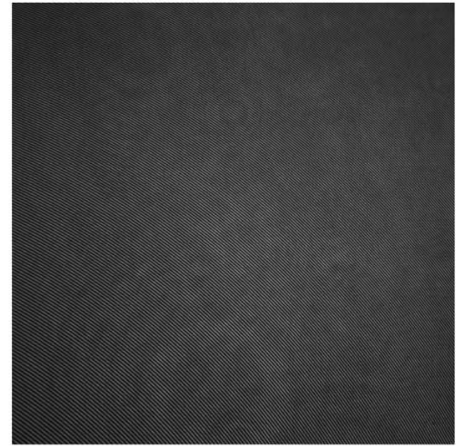

(b)

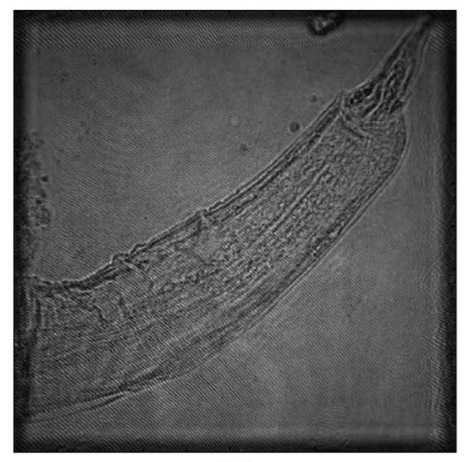

(e)

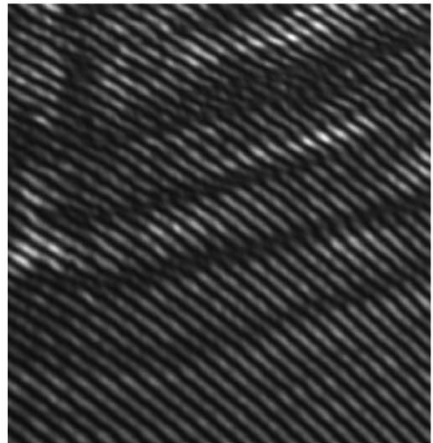

(c)

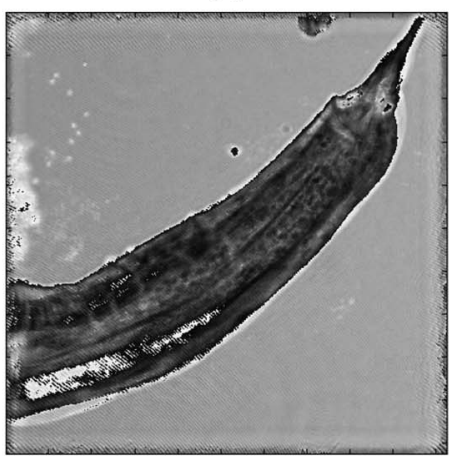

(f)

Fig. 13. Riesz demodulation performance on C. elegans holograms. (a) Hologram of C. elegans, (b) reference hologram, (c) zoomed-in part of the hologram (approximately 1/16th the size of the hologram from the lower left corner), (d) zoomed-in part of the reference hologram (approximately $1 / 16$ the size of the reference hologram from the lower left corner), (e) amplitude-contrast image , and (f) phase-contrast image (wrapped phase). The orientation map is estimated with a Gaussian kernel of standard deviation $\sigma=5$. 
images obtained are shown to be consistent in terms of quantitative values, as it has been possible to focus the wavefronts through digital propagation $(d=7 \mathrm{~cm})$, leading to the presented results. The fine structures in the specimen can be identified both in amplitude and phase, with a spatial resolution that is consistent with the aperture of the employed optical system.

\section{CONCLUSIONS}

We addressed the problem of hologram demodulation for the general case of nonplanar fringes. We first considered the 1D demodulation problem and then systematically developed a 2D counterpart by employing the complex Riesz transform, which is a steerable extension of the Hilbert transform in higher dimensions. The interesting aspect is the link between the Riesz transform and the directional Hilbert transform. By invoking quasi-eigenfunction approximations, we showed how quadratures may be derived from the Riesz transform. The ability of the Riesz transform to yield quadratures by compensating for the local changes of orientation is crucial to the success of demodulation. Interestingly, the proposed framework is self-sufficient because the orientation also can be estimated using the complex Riesz transform. The proposed approach requires a onetime calibration to obtain a reference hologram, which corresponds to an empty field of view in the object arm. Experiments on simulated as well as real holograms of $C$. elegans showed that the proposed technique effectively handles nonplanar fringes.

\section{ACKNOWLEDGMENTS}

The work is supported in part by the Swiss National Science Foundation (SNSF) grant 205320-130543, by European Research Council (ERC) grant ERC-2010-AdG 267439-FUN-SP, and by the Center for Biomedical Imaging of the GenevaLausanne Universities and Ecole Polytechnique Fédérale de Lausanne (EPFL) (CIBM). C. S. Seelamantula also acknowledges support of the Indian Institute of Science new faculty startup grant (14-0403-0012-01) and the Department of Science and Technology (DST)-Intensive Research in High Priority Areas (DST-IRHPA) project DSTO-943.

\section{REFERENCES}

1. S. Haykin, Communication Systems, 4th ed. (Wiley, 2001).

2. D. Gabor, "Theory of communication," J. IEE Part 3 93, 429-457 (1946).

3. A. Papoulis, Signal Analysis (McGraw-Hill, 1977).

4. A. Papoulis, Systems and Transforms With Applications in Optics (Krieger, 1981).

5. E. Stein and G. Weiss, Introduction to Fourier Analysis on Euclidean Spaces (Princeton University, 1971).

6. S. L. Hahn, "Multidimensional complex signals with singleorthant spectra,” Proc. IEEE 80, 1287-1300 (1992).

7. W. T. Freeman and E. H. Adelson, "The design and use of steerable filters," IEEE Trans. Pattern Anal. Machine Intell. 13, 891-906 (1991).
8. M. Unser and D. Van De Ville, "Wavelet steerability and the higher-order Riesz transform,” IEEE Trans. Image Process. 19, 636-652 (2010).

9. M. Unser, D. Sage, and D. Van De Ville, "Multiresolution monogenic signal analysis using the Riesz-Laplace wavelet transform," IEEE Trans. Image Process. 18, 2402-2418 (2009).

10. T. Bülow and G. Sommer, "Hypercomplex signals-a novel extension of the analytic signal to the multidimensional case," IEEE Trans. Signal Process. 49, 2844-2852 (2001).

11. K. G. Larkin, D. J. Bone, and M. A. Oldfield, "Natural demodulation of two-dimensional fringe patterns. I. General background of the spiral phase quadrature transform,” J. Opt. Soc. Am. A 18, 1862-1870 (2001).

12. K. G. Larkin, "Natural demodulation of two-dimensional fringe patterns. II. Stationary phase analysis of the spiral phase quadrature transform,” J. Opt. Soc. Am. A 18, 1871-1881 (2001).

13. M. Felsberg and G. Sommer, "The monogenic signal," IEEE Trans. Signal Process. 49, 3136-3144 (2001).

14. T. Colomb, E. Cuche, F. Charriere, J. Kuhn, N. Aspert, F. Montfort, P. Marquet, and C. Depeursinge, "Automatic procedure for aberration compensation in digital holographic microscopy and applications to specimen shape compensation," Appl. Opt. 45, 851-863 (2006).

15. M. Liebling, T. Blu, and M. Unser, "Complex-wave retrieval from a single off-axis hologram,” J. Opt. Soc. Am. A 21, 367-377 (2004).

16. C. S. Seelamantula, N. Pavillon, C. Depeursinge, and M. Unser, "Exact complex-wave reconstruction in digital holography," J. Opt. Soc. Am. A 28, 983-992 (2011).

17. J. P. Havlicek, D. S. Harding, and A. C. Bovik, "Multidimensional quasi-eigenfunction approximations and multicomponent AM-FM models," IEEE Trans. Image Process. 9, 227-242 (2000).

18. J. W. Goodman and R. W. Lawrence, "Digital image formation from electronically detected holograms," Appl. Phys. Lett. 11, 77-79 (1967).

19. E. Cuche, P. Marquet, and C. Depeursinge, "Simultaneous amplitude-contrast and quantitative phase-contrast microscopy by numerical reconstruction of Fresnel off-axis holograms," Appl. Opt. 38, 6994-7001 (1999).

20. E. Cuche, P. Marquet, and C. Depeursinge, "Spatial filtering for zero-order and twin-image elimination in digital off-axis holography," Appl. Opt. 39, 4070-4075 (2000).

21. P. Ferraro, G. Coppola, S. D. Nicola, A. Finizio, and G. Pierattini, "Digital holographic microscope with automatic focus tracking by detecting sample displacement in real time," Opt. Lett. 28, 1257-1259 (2003).

22. T. Colomb, J. Kühn, F. Charrière, C. Depeursinge, P. Marquet, and N. Aspert, "Total aberrations compensation in digital holographic microscopy with a reference conjugated hologram," Opt. Express 14, 4300-4306 (2006).

23. J. W. Head and C. G. Mayo, "The response of a network to a frequency-modulated input voltage," Proc. IEE C 305R, 509-512 (1958).

24. E. Bedrosian, "A product theorem for Hilbert transforms," Proc. IEEE 51, 868-869 (1963).

25. A. H. Nuttall and E. Bedrosian, "On the quadrature approximation to the Hilbert transform of modulated signals," Proc. IEEE 54, 1458-1459 (1966).

26. H. Urkowitz, "Hilbert transforms of band-pass functions," Proc. IRE 50, 2143 (1962).

27. G. H. Golub and C. F. V. Loan, Matrix Computations, 3rd ed. (Johns Hopkins University, 1996). 